\title{
Adjoint Equations and Error Measure in Radiative Heat Transfer with Diffuse Reflection
}

\author{
Pierre E. Vueghs* \\ Doctor \\ Aerospace \& Mechanical Engineering Department \\ University of Liège \\ Liège, Belgium B4000 \\ Email: Pierre.Vueghs@ulg.ac.be \\ Pierre Rochus \\ Professor \\ Liège Space Center \\ University of Liège \\ Liège, Belgium B4000 \\ Email:PRochus@ulg.ac.be
}

In this paper, we develop an application of the importance equation (which is an adjoint equation of the radiosity equation) in the case of isothermal, diffuse surfaces. We recall the formulation of the radiosity equation, in function of the nature of the boundary condition (either known temperature or fixed radiative heat flux). We define the importance as the quantity dual to radiosity. We explain how these equations can be used after the resolution of a radiative heat transfer situation, as a post processing step, to establish the accuracy of each individual radiative link between the active faces of a tri-dimensional surface geometrical model.

\section{Background}

In order to design the thermal control system of a space mission, the thermal engineer often uses dedicated software. As the radiative component can be predominant, software is very often based on Monte Carlo ray tracing to compute the energy exchanges between the surfaces which compose the geometrical model, as well as the heat fluxes from the heat sources to the spacecraft and the evacuation of heat to the deep space. The accuracy of Monte Carlo ray tracing is function of the number of traced rays; this number of rays is left to the discretion of the engineer. A bad estimate may lead to an unacceptable error or an unnecessary computation load. In this paper, we propose a way to compute the energy error associated with each surface. It allows us to identify the less accurate surfaces, which could require additional rays to be traced. It is a first step to a statistical accuracy control, which could automatically compute the number of rays to achieve the required level of accuracy.

\footnotetext{
${ }^{*}$ Address all correspondence to this author
}

\section{Introduction}

The energy balance of a radiative situation can be computed by solving a set of equations, based on radiosity. The propagation of radiosity through the geometrical model is governed by a transport operator $\mathcal{T}$, based on geometrical view factors.

Using a stochastic ray tracing algorithm to compute the view factors, a discrete approximation to the exact transport operator is obtained. A geometrical error can be computed for each individual view factor. This error is purely geometrical and does not take into account the energy exchanges through the model.

The purpose of this paper is to present a method based on the notion of importance and adjoint equations of the radiosity system to establish an energy measure of the error induced by the radiative computation. Based on a first measure of the geometrical error (which affects the view factors), the adjoint equations can be used to obtain a measure of the energy error, which affects heat transfer.

The notion of importance has first been defined in image synthesis, to accelerate the generation of realistic images where light experiences multi-reflections [1]. Importance is used to identify the radiative interactions which have the highest impact on the distribution of light, as it is seen from the observer (i.e. a pinhole camera, the eye, a radiometer).

In radiative heat transfer, there is no such viewdependent aspect; each surface has influence on all other surfaces, either by direct or indirect radiation. However, the characterization of important radiative interactions is of great interest for the thermal engineer. Contrary to classical applications in image synthesis, which aim to accelerate the 
generation of pictures, the notions of importance and adjoint equations are extended in radiative heat transfer in order to give a measure of the energy error induced by the radiative solution.

The computation of an energy error, obtained after the resolution of the radiosity equation and the determination of heat fluxes and temperatures, is an innovative development in the field of radiative heat transfer; it could yield a clear improvement for software dedicated to space thermal engineering. In this work, for reasons of simplicity, we only consider diffuse, isothermal patches, characterized by constant emittance $\varepsilon$ and diffuse reflectance $\rho$.

\section{Importance}

The notion of importance has been intensively used in rendering in order to accelerate the generation of realistic images [2]. The approach is based on two dual physical quantities: radiosity and importance. The distributions of these two quantities are governed by a radiative transport operator $\mathcal{T}$ which is based on the notion of view factors.

\subsection{View factors and geometrical error}

In radiative heat transfer, the exchanges of energy are governed by an adimensional number called view factor. The diffuse view factor $F_{i-j}$ is defined as the fraction of the radiative energy diffusely emitted by a patch $P_{i}$ which directly strikes a patch $P_{j}$ :

$$
F_{i-j}=\frac{1}{A_{i}} \int_{A_{i}} \int_{A_{j}} \frac{\cos \left(\theta_{i}\right) \cos \left(\theta_{j}\right)}{\pi s_{i-j}^{2}} V_{i-j} d A_{j} d A_{i}
$$

where the point $d A_{i}$ on the patch $P_{i}$ is located by the vector $\overrightarrow{s_{i}} ; \overrightarrow{s_{i-j}}=\overrightarrow{s_{j}}-\overrightarrow{s_{i}}$ is the vector joining the point $d A_{i}$ and a point $d A_{j}$ on the patch $P_{j} ; \theta_{i}$ is the angle between the vector $\overrightarrow{s_{i-j}}$ and the local normal $\overrightarrow{n_{i}}$ of the surface $A_{i}$ at the point $d A_{i} ; V_{i-j}$ is the visibility function, equal to 1 when the two points $\overrightarrow{s_{i}}$ and $\overrightarrow{s_{j}}$ can see each other, equal to 0 otherwise. This last factor is responsible for discontinuities of the kernel when it is integrated on the surface $A_{j}$. This increases the difficulty of the computation.

A way to compute view factors is stochastic ray tracing. It is a robust and reliable method, which can be used in order to model a large panel of surface phenomenons, such as reflection and transmission, as well as volume phenomenons, such as diffusion. In this work, we only consider diffuse reflection but the extension to specular reflection and other surface phenomenons will be considered in further work.

Another advantage of stochastic ray tracing is that the accuracy of the random process can be defined by two parameters: the maximum relative error $\varepsilon$ and the confidence interval $\alpha$. In function of the configuration (i.e. the view factor), the necessary number of rays can be established in order to compute the view factor within the desired accuracy [3]. This is a measure of the geometrical error which affects the view factors.
In this work, we used the stratified hemisphere method to generate the direction of the rays, to compute the view factors. This method is characterized by a convergence superior to the classical one [4]: the relative error is inversely proportional to $N^{\frac{3}{4}}$, where $N$ is the total number of rays traced from patch $P_{i}$. In this case, the absolute error associated with the view factor $F_{i-j}$ is given by the following relation:

$$
\Delta F_{i-j}=\varepsilon F_{i-j}=\operatorname{erf}^{-1}(\alpha) \frac{\sqrt{2 d}}{N^{\frac{3}{4}}}
$$

where $\operatorname{erf}^{-1}$ refers to the inverse of the error function, and $d$ is a geometrical parameter, studied in [5]. If the Monte Carlo method is used in this paper (because it easily gives a measure of the geometrical error), the notions of importance and adjoint equations developed in this paper are not limited to this method and can be used with any other method for the computation of the view factors.

The computation of the view factors is the most time consuming step of the thermal solution. We can also assess that it is the main source of errors in the complete solution. Therefore, the impact of this error on the thermal results has to be estimated. This is the purpose of this paper.

\subsection{Radiosity equation}

In this Section, the expression of the radiosity equation is recalled, taking into account the boundary conditions. The thermal radiosity $J_{i}$ of a surface $i$ is defined as the sum of the self-emitted flux $\varepsilon_{i} E_{b, i}$, and the diffuse reflection of the incoming radiation $H_{i}[6]$ :

$$
J_{i}=\varepsilon_{i} E_{b, i}+\rho_{i} H_{i}
$$

Following [6], the radiative balance of the thermal model can be expressed by the following set of equations, based on the radiosities $J_{j}$ and the previously defined view factors $F_{i-j}$ :

$$
\frac{q_{i}}{\varepsilon_{i}}=E_{b, i}-\sum_{j=1}^{N} F_{i-j} J_{j}-H_{0, i} \quad \forall i \in[1, N]
$$

The radiative heat flux $q_{i}$, the self-emitted power $E_{b, i}$ and the radiosity $J_{i}$ of a patch $P_{i}$ are linked by the following relation:

$$
\frac{q_{i}}{\varepsilon_{i}}=\frac{1}{1-\varepsilon_{i}}\left(E_{b, i}-J_{i}\right)
$$

For each patch $P_{i}$, two expressions can be obtained, in function of the nature of the boundary condition. Let us assume that the temperatures of the $n$ first patches are known while the corresponding heat fluxes are unknown. On the other hand, the radiative heat fluxes of the $N-n$ other 
patches are fixed, and their temperatures have to be computed. For each patch, relations (4) and (5) are combined to remove the unknown quantity. The following relations are obtained:

$$
\begin{aligned}
& J_{i}=\varepsilon_{i} E_{b, i}+\rho_{i} H_{0, i}+\rho_{i} \sum_{j=1}^{N} F_{i-j} J_{j} \quad \forall i \in[1, n] \\
& J_{i}=q_{i}+H_{0, i}+\sum_{j=1}^{N} F_{i-j} J_{j} \quad \forall i \in[n+1, N]
\end{aligned}
$$

The set of radiosity equations can be rewritten as follows:

$$
\mathcal{T} J=S
$$

where the matrix $\mathcal{T}$ is called the transport operator and is equal to:

$$
\mathcal{T}=\left(\begin{array}{ccccc}
1-\rho_{1} F_{1-1} & \ldots & -\rho_{1} F_{1-n} & \ldots & -\rho_{1} F_{1-N} \\
\vdots & \ddots & \vdots & \ddots & \vdots \\
-\rho_{n} F_{n-1} & \ldots & 1-\rho_{n} F_{n-n} & \ldots & -\rho_{n} F_{n-N} \\
-F_{n+1-1} & \ldots & -F_{n+1-n} & \ldots & -F_{n+1-N} \\
\vdots & \ddots & \vdots & \ddots & \vdots \\
-F_{N-1} & \ldots & -F_{N-n} & \ldots & 1-F_{N-N}
\end{array}\right)
$$

The thermal source $S$ is based on the boundary conditions and the external irradiation; this vector is assumed to be exact. Relation (8) will be referred as the thermal radiosity equation.

Transport operator $\mathcal{T}$ is a discrete approximation of the real, continuous transport operator. It is a matrix of real numbers; this implies that $\mathcal{T}^{*}=\mathcal{T}^{T}$. For the patches with fixed heat flux, the diffuse reflectance does not appear explicitly. At the level of the transport operator, these surfaces can be considered as ideal, diffuse reflectors, associated with a reflectance equal to $100 \%$ (of course, once the thermal radiosities have been computed, the real emittances have to be used with equation (5) to obtain the corresponding absolute temperatures). In order to homogenize the expression of the transport operator, a fictive model is defined, where the diffuse reflectances of the patches with fixed heat flux are set to $100 \%$. If $\rho^{*}$ denotes the new vector of diffuse reflectance, the transport operator is based on the view factor matrix $\mathcal{F}$ :

$$
\mathcal{T}=I_{N}-\rho^{*} \mathcal{F}
$$

where $I_{N}$ denotes the identity matrix of $\operatorname{rank} N$.

\subsection{Adjoint equation and definition of importance}

In this work, the adjoint equations are used to measure the radiative error induced by the approximation of the transport operator $\mathcal{T}$. A quantity, dual to radiosity, noted $I$, and called importance, is defined by the following relation [7]:

$$
\mathcal{T}^{*} I=R
$$

where the operator $\mathcal{T}^{*}$ is the adjoint of the transport operator $\mathcal{T}$, and $R$ is the initial importance.

In radiative heat transfer, each point $d A_{i}$ of each patch $P_{i}$ must be considered for the solution of the radiative situation. If each point is associated with an unit initial importance, the initial importance $R_{i}$ of patch $P_{i}$ is equal to its area $A_{i}$ :

$$
R_{i}=A_{i} \quad \forall i \in[1, N]
$$

Importance could be seen as the dual quantity of radiative heat flux, transported like heat flux but in the opposite direction, in a fictive model where the surfaces with fixed heat flux are characterized by a diffuse reflectance of $100 \%$. $\rho^{*}$ denoting the reflectance vector of this fictive model, the distribution of importance is governed by the following relation:

$$
I_{i}=A_{i}+\sum_{j=1}^{N} \rho_{j}^{*} F_{j-i} I_{j}
$$

\subsection{Inner product and duality}

On the basis of the radiosity- and initial importancevectors, we can compute the following inner product, initially defined in image synthesis [7], where each individual term represents the radiative energy emitted by a patch $P_{i}[2]$ :

$$
v(J)=R^{T} J=\left(\mathcal{T}^{T} I\right)^{T} J=I^{T} \mathcal{T} J=I^{T} S
$$

This relation highlights the duality of radiosity and importance. $v(J)$ is called the global radiative energy.

\subsection{Error analysis}

The geometrical model has been discretized into $N$ patches, i.e. into $N$ surfaces of finite area. In our algorithm, the view factors are computed by stochastic ray tracing. As the number of rays is limited, the view factors are approximated. An error measure can be derived for each single view factor $[3,4]$. This error measure is only geometrical; it is not representative of the energy error. Here, a way to derive such an error measure is presented. Let $\tilde{\mathcal{T}}$ be the approximation of the transport operator $\mathcal{T}$, obtained by ray tracing:

$$
\tilde{\mathcal{T}}=\mathcal{T}+\Delta \mathcal{T}
$$

The error is assumed to be due to the view factors only. Each term $\tilde{\mathcal{T}}_{i-j}$ of the transport operator is affected by an error $-\rho_{i}^{*} \Delta F_{i-j}$, where $\rho^{*}$ refers to the reflectance of the fictive 
model. The thermal radiosity, computed by equation (8), is also approximated:

$$
\widetilde{J}=J+\Delta J
$$

It is an acceptable hypothesis to suppose that the source term $S$ is exactly known. Equation (8) then yields the following relation:

$$
\widetilde{\mathcal{T}} \widetilde{J}=S
$$

By combining equations (15) and (17), the following expression is obtained, where the operator is the exact transport operator $\mathcal{T}$ and where the source term is perturbed by a quantity $\Delta \mathcal{T} \widetilde{J}$ :

$$
\mathcal{T} \widetilde{J}=S-\Delta \mathcal{T} \widetilde{J}
$$

The energy error is defined thanks to the $v$-function:

$v(J-\widetilde{J})=R^{T}(J-\widetilde{J})=R^{T} J-R^{T} \widetilde{J}=I^{T} S-I^{T} \mathcal{T} \widetilde{J}=I^{T} \Delta \mathcal{T} \widetilde{J}$

The quantity $I^{T} \Delta \mathcal{T} \widetilde{J}$ is the error introduced in the global radiative energy $v(J)$ by the approximation of the transport operator $\mathcal{T}$ and the radiosities $\widetilde{J}$. The importance is obtained by solving equation (13). As importance is also affected by the approximation of the transport operator, $\widetilde{I}^{T} \Delta \mathcal{T} \widetilde{J}$ is used as the approximation of $I^{T} \Delta \mathcal{T} \widetilde{J}$. Equation (19) is a double sum on all the surfaces which compose the geometrical model. A particular term $\widetilde{I}_{i} \Delta \mathcal{T}_{i-j} \widetilde{J}_{j}=\widetilde{I}_{i} \rho_{i}^{*} \Delta F_{i-j} \widetilde{J}_{j}$ corresponds to the error characterizing the energy link from surface $j$ to surface $i$. This expression allows us to establish a measure of the error induced by the approximation of the transport term $\mathcal{T}_{i-j}$. From the expression of the transport operator (10), supposing that the error is linked to the computation of the view factors, each term $\mathcal{T}_{i-j}$ of the transport operator is affected by an error $-\rho_{i}^{*} \Delta F_{i-j}$.

\section{Application}

The method has been used to solve the case of a solar collector with diffuse surfaces, inspired from [6]. The geometrical configuration is shown in Figure 1. The boundary conditions are given in Figure 2. The collector is characterized by an emittance of $80 \%$ and a diffuse reflectance of $20 \%$ while the mirror's emittance is $10 \%$ and the mirror's diffuse reflectance is equal to $90 \%$. The geometrical model is associated with a finite element mesh [8], as it has been developed in the framework of the thesis [5].

\subsection{View factors}

The first step consists in computing the view factors (see Figure 3). This is done by a ray tracing process based on the stratified hemisphere method [4], accelerated with the parametric method [9]. If a two-node model is considered, the

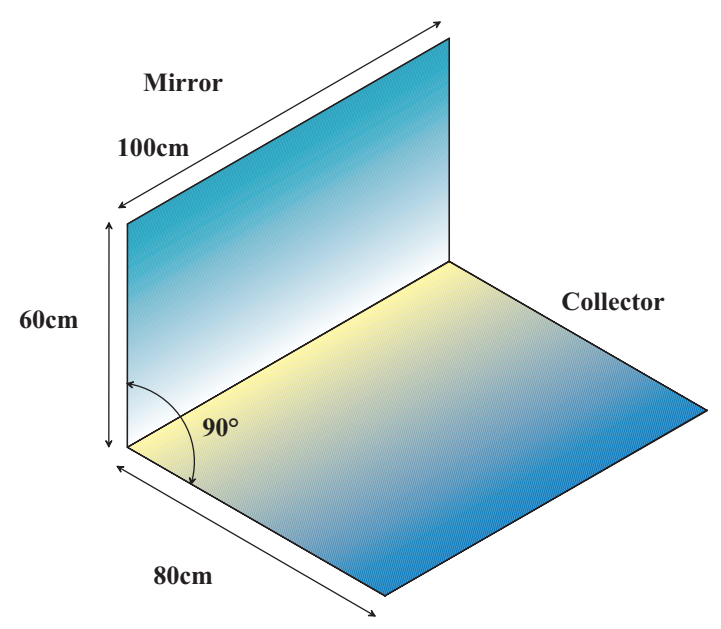

Fig. 1. Geometrical configuration.

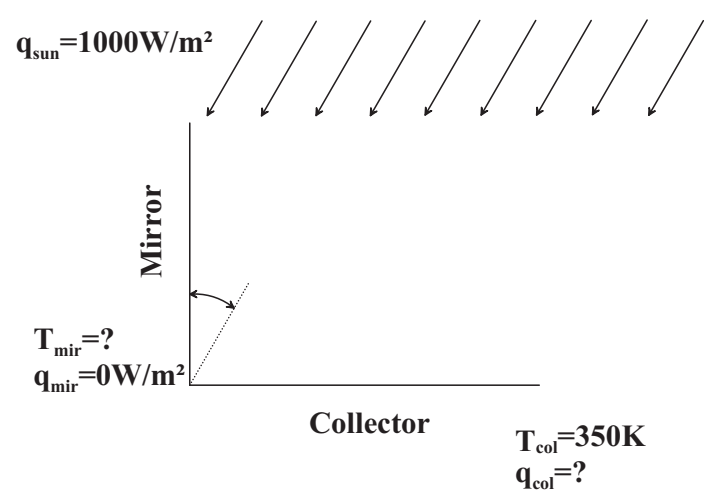

Fig. 2. Boundary conditions.

obtained view factor matrix is given as follows (number 1 refers to the collector, number 2 is the mirror):

$$
F=\left[\begin{array}{cc}
0 & 0.1910 \\
0.2547 & 0
\end{array}\right]
$$

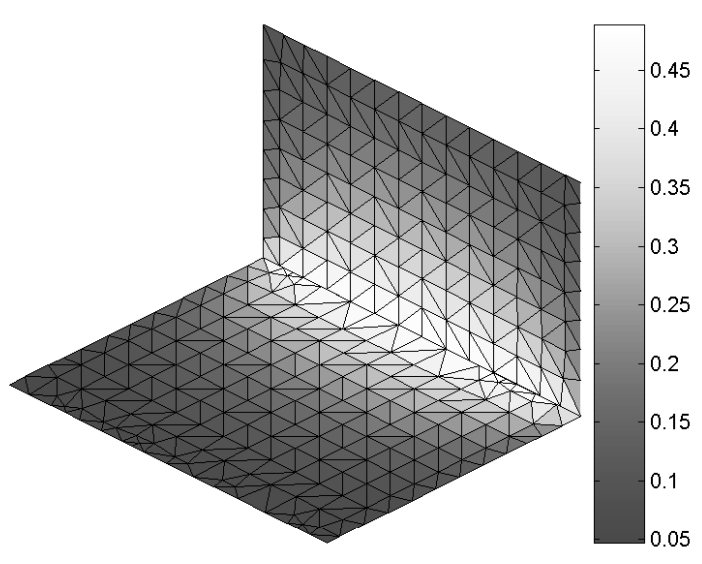

Fig. 3. View factors.

The absolute errors (estimated by the stratified hemi- 
sphere method [4]) affecting the view factors are given as follows:

$$
\delta F_{1-2}=1.60110^{-3} \quad \delta F_{2-1}=1.78310^{-3}
$$

\subsection{Temperatures and radiative heat fluxes}

The second step consists in solving the radiative thermal problem, given a set of boundary conditions. The computed temperatures are displayed in Figure 4 while the heat fluxes are given in Figure 5.

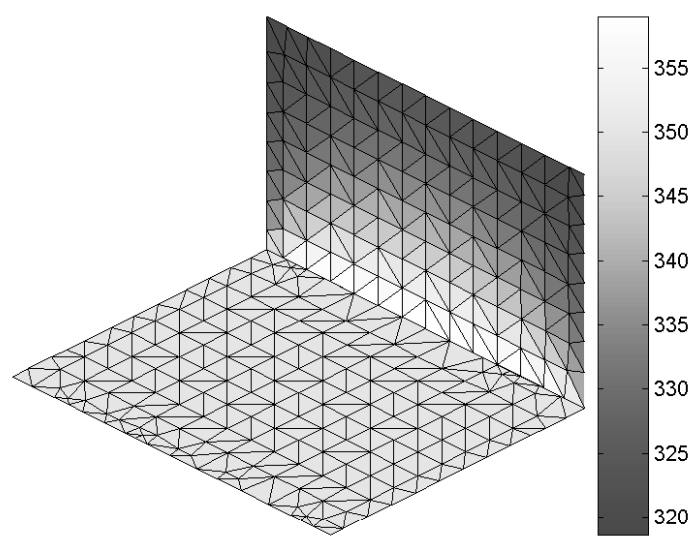

Fig. 4. Temperature distribution.

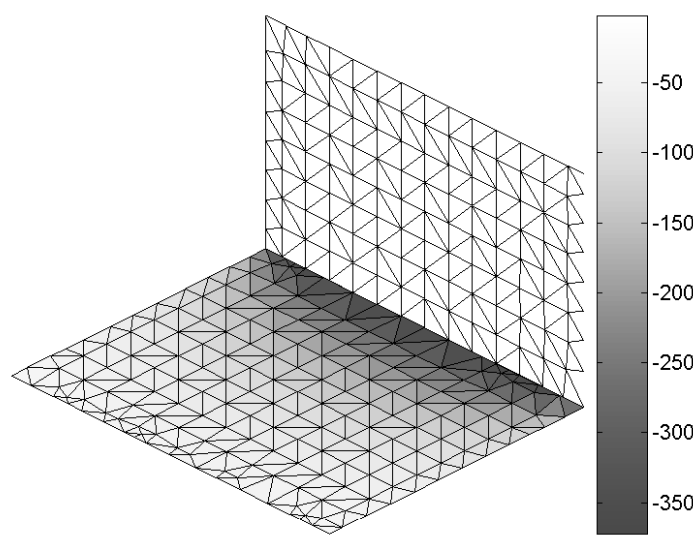

Fig. 5. Heat flux distribution.

\subsection{Thermal radiosities}

Based on the temperatures and the thermal heat fluxes, relation (5) is used to compute the corresponding thermal radiosities (see Figure 6). This is the third step of the error computation process.

\subsection{Importance}

The last quantity to compute is the importance. The initial importance vector is defined by the area of the elements.

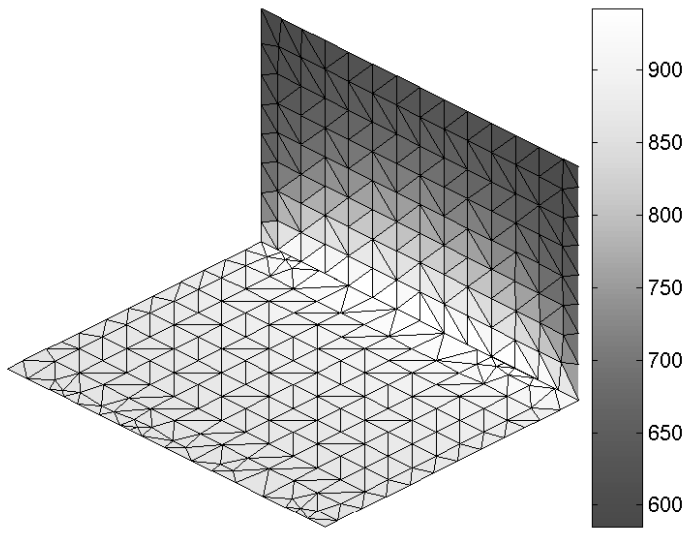

Fig. 6. Radiosity distribution.

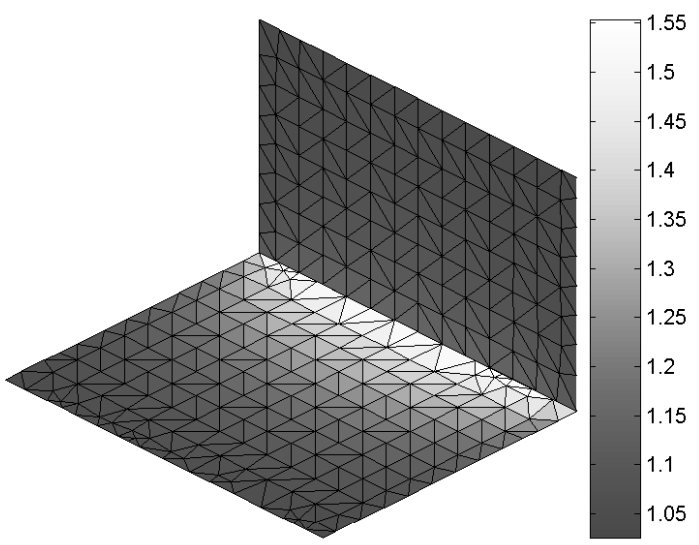

Fig. 7. Surface importance.

In function of the view factors and the surface thermo-optical properties, we obtain the distribution of importance given in Figure 7 (the displayed quantity is in fact the importance per unit area). This surface importance is always larger than (or equal to) unity.

\subsection{Error measure}

On the basis of the stratified hemisphere method [4], we are able to compute the absolute error affecting the view factors between the two surfaces (equation (21)). This absolute error is introduced in the expression of the energy error. This error has to be compared with the radiative energy $v(J)$, which is equal to $1143.8 \mathrm{~W}$. The relative energy errors associated with each link are given as follows, by the quantity $\left.\delta Q_{i-j}\right|_{\varepsilon}=\widetilde{I}_{i} \rho_{i} \delta F_{i-j} \widetilde{J}_{j}$.

The relative errors affecting the energy exchanges between the surfaces are:

$$
\left.\delta Q_{1-2}\right|_{\varepsilon}=\left.1.966710^{-4} \quad \delta Q_{2-1}\right|_{\varepsilon}=8.246410^{-4}
$$

The surface associated with large view factor error is not necessarily associated with the largest radiative error. The geometrical errors which characterize view factors have to be weighted by the radiosity of the surfaces, the importance 
and reflectance. The radiosities of the two surfaces are similar, as well as their importance. The geometrical error characterizing the view factors are also of the same magnitude. In this problem, the main radiative link is from the mirror to the collector, because of the larger reflectance of the mirror. The weak reflectance of the collector reduces the impact of the other link on the radiative energy $v(J)$. Due to this consideration, it appears that the link "mirror-to-collector" has to be carefully computed during the ray tracing process. The computation effort has to be focused on this link.

\section{Conclusions and perspectives}

In this paper, we have established the basis of a formulation using the adjoint equations for radiosity and importance, which can be used in a post process step in order to estimate the error characterizing each radiative link in a 3D geometrical model. The adjoint equations are not used to accelerate the computation of the radiative equilibrium, as is done in rendering, but to yield guarantees on the accuracy of the thermal results. Nevertheless, a first, coarse temperature distribution should allow us to estimate the thermal radiosities and to use the full potential of the adjoint equations, such as an active control of the radiative error. This active control of the accuracy could be used during the computation of the view factors in order to accelerate this time-consuming process.

In this document, we limit ourselves to diffuse, isothermal surfaces. A next step could be to include specular and glossy reflection, as well as non-isothermal surfaces.

The introduction of other modes of heat transfer should also be addressed. If the thermal situation is essentially governed by heat radiation, the approach presented in this paper is valid. If conduction is introduced, it alters the temperature distribution and then the radiosity distribution. If the problem is mainly governed by conduction, our approach is no longer valid. A careful study should establishes the validity of our error measure when conductive heat transfer is also present.

\section{Achnowledgements}

This work has been supported by the Belgian National Fund for Scientific Research (FNRS) and the European Space Agency (ESA/ESTEC), contract 20180/06/NL/PA

\section{References}

[1] Pattanaik, S. N., and Mudur, S. P., 1995. “Adjoint equations and random walks for illumination computation". ACM Transactions on Graphics, 14(1), January, pp. 77102.

[2] Christensen, P. H., 2003. "Adjoint and importance in rendering: An overview". IEEE Transactions on Visualization and Computer Graphics, 9(3), pp. 329-340.

[3] Planas, P., 1993. "Statistical error control for radiative software based on monte carlo methods". Proceed- ings of the 23rd ICES Conference, SAE Technical Paper 932258.

[4] Vueghs, P., de Koning, H. P., Pin, O., and Beckers, P., 2008. "Random hemisphere method for radiation ray tracing computations". In Eurotherm 2008 - 5th European Thermal-Sciences Conference, A. A. S. G. G. M. Stoffels, T. H. van der Meer, ed.

[5] Vueghs, P. E., 2008-2009. "Innovative Ray Tracing Algorithms for Space Thermal Analysis". PhD thesis, University of Liège.

[6] Modest, M. F., 2003. Radiative Heat Transfer, second edition. Academic Press.

[7] Smits, B. E., Arvo, J. R., and Salesin, D. H., 1992. "An importance-driven radiosity algorithm". Computer Graphics (ACM SIGGRAPH 92 Proceedings), 26(4), July, pp. 273-282.

[8] Chin, J. H., Panczak, T. D., and Fried, L., 1989. "Finite element and raytracing in coupled thermal problems". In Sixth International Conference on Numerical Methods in Thermal Problems, P. Press, ed., pp. 683-701.

[9] Vueghs, P., de Koning, H. P., Pin, O., and Beckers, P., 2008. "Use of geometry in finite elements thermal radiation combined with ray tracing". In Fourth International Conference on Advanced COmputational Methods in ENgineering (ACOMEN 2008), M. Hogge, R. V. Keer, L. Noels, L. Stainier, J.-P. Ponthot, J.-F. Remacle, and E. Dick, eds., University of Liege, Belgium. 